\title{
A Countable System of Fractional Inclusions with Periodic, Almost, and Antiperiodic Boundary Conditions
}

\author{
Ahmed Salem (iD ${ }^{1}$ and Aeshah Al-Dosari ${ }^{1,2}$ \\ ${ }^{1}$ Department of Mathematics, Faculty of Science, King Abdulaziz University, P.O. Box 80203, Jeddah 21589, Saudi Arabia \\ ${ }^{2}$ Prince Sattam University, Al-Kharj 16278, Saudi Arabia \\ Correspondence should be addressed to Ahmed Salem; ahmedsalem74@hotmail.com
}

Received 22 March 2021; Accepted 28 May 2021; Published 14 June 2021

Academic Editor: Cristiana J. Silva

Copyright (c) 2021 Ahmed Salem and Aeshah Al-Dosari. This is an open access article distributed under the Creative Commons Attribution License, which permits unrestricted use, distribution, and reproduction in any medium, provided the original work is properly cited.

\begin{abstract}
This article is dedicated to the existence results of solutions for boundary value problems of inclusion type. We suggest the infinite countable system to fractional differential inclusions written by $\mathrm{ABC}^{\alpha} D^{\alpha}\left[v_{i}(t)\right] \in \mathscr{Y}_{i}\left(t,\left\{v_{i}(t)\right\}_{i=1}^{\infty}\right)$. The mappings $y_{i}\left(t,\left\{v_{i}(t)\right\}_{i=1}^{\infty}\right)$ are proposed to be Lipschitz multivalued mappings. The results are explored according to boundary condition $\sigma \nu_{i}(0)=\gamma v_{i}(\rho), \quad \sigma, c \in \mathbb{R}$. This type of condition is the generalization of periodic, almost, and antiperiodic types.
\end{abstract}

\section{Introduction}

Consider the following infinite system:

$$
\begin{gathered}
\mathrm{ABC}^{\alpha}\left[\nu_{i}(t)\right] \in y_{i}\left(t,\left\{v_{j}(t)\right\}_{j=1}^{\infty}\right), \quad i \in \mathbb{N}, t \in[0, \rho], \\
\sigma v_{i}(0)=\gamma v_{i}(\rho), \quad \sigma, \gamma \in \mathbb{R},
\end{gathered}
$$

where ${ }_{\mathrm{ABC}} D^{\alpha}$ denotes the Atangana-Baleanu fractional derivative in the Caputo sense of order $\alpha \in(0,1]$ and $\left\{y_{i}\right\}_{i \in \mathbb{N}}$ is an infinite countable family of Lipschitz continuous multivalued mappings. This means there is an infinite countable sequence of continuous real-valued functions $\left\{v_{i}(t)\right\}_{i \in \mathbb{N}}$ satisfying problems (1) and (2). In this case, we can define the function $V(t):[0, \rho] \longrightarrow \mathbb{R}^{\mathbb{N}}$ by $V(t)=\left(\nu_{i}(t)\right)_{i \in \mathbb{N}}$. This function denotes the sequence of solutions for the given system.

In the field of infinite systems, the research to fractional differential problems started via ordinary derivatives (see [1-4] and the mentioned references therein). Then, many scholars were attracted to develop these problems into the ones associated with fractional derivatives. For instance, see the required results in [5-8] and references cited therein. The importance of the infinite system was arising naturally in the description of physical problems such as stochastic (stochastic metapopulation) models $[9,10]$, models descried by the Becker-Döring cluster $[11,12]$, and optimal pursuit equations [13] and the control problems for the models descried by parabolic and hyperbolic equations [14].

The concept of fractional derivative arose before 300 years when L'Hospital asked in 1695 which is addressed in Leibnitz notation for the $n$th derivative "What would happen if the order $n=1 / 2$ ?". So, the idea of fractional derivative started by operators with power kernel (Riemman and Caputo derivatives). It has been industrialized due to complexities associated with the heterogeneous phenomenon. The fractional differential operators are capable of capturing the behavior of multifaceted media as they have diffusion processes. In this field, many researchers have paid attention in several ways to develop these derivatives. For instance, they found the ways for the development to new ones without the problem of nonsingularity (Caputo-Fabrizio with the exponential kernel) and then without nonlocality (Atangana-Baleanu with the Mittag-Leffler kernel). Mittag-Leffler kernel in the AB derivative helps to understand the beginning and the end of a considered phenomenon which is due to the memory effect of the Mittag-Leffler function. Additionally, in some works, it was proved that the $\mathrm{AB}$ derivative can generate chaotic behaviors in some linear and nonlinear systems for certain values taken 
by the derivative order. In other situations, some researchers have shown that the fractional derivatives lost some of the basic properties that usual derivatives have such as the product rule and the chain rule. For the sake of solving this problem, they investigated the conformable fractional derivative. This derivative is important to develop the Lie symmetry analysis for differential equations involving different fractional derivatives such as the Caputo-Fabrizio derivative and Atangana-Baleanu derivative. In fact, the development of fractional calculus theory matches with the development of analytical and numerical methods for solving fractional differential equations and systems. For showing this importance, we refer to see [15-18] and the references therein.

In [19], we studied how to generate the differential equations and inclusions by one form (we call them as equiinclusion problems). Then, we studied the solvability of this form. Next, in [20], we generated the fractional differential equation at resonance on the half line into the inclusion one and explored the existence results of positive solutions for this problem. After research and reading about the infinite system topic, we find that all proven treatises are linked only to single-valued operators. So, what we refer here is to survey the infinite fractional differential system proposed with multivalued mappings. This means we hatch a generalization of previous literature studies in the infinite system field. This draws a way to new and important extents for the infinite system theory of nonlinear analysis. Furthermore, it would be useful to express some descriptions of complicated physical phenomena. We have a strong anticipation that the partial differential inclusion which leads to the infinite system of fractional differential inclusions would be very influential to make a fundamental shift in the theory of complicated neural sets, huge and stochastic branching processes, and the theory of dissociation of polymers. Also, by the inclusion system with ${ }_{\mathrm{ABC}} D^{\alpha}$, we think that modeling and computations will be performed to explore deep and manifold aspects of mixed convective flow of nanofluids and random flow processes of so many fluids.

Our work is concerned with the existence of antiperiodic, periodic, and almost periodic solutions for problem (1) in the Banach space $\ell_{p}\left(\mathbb{R}^{\mathbb{N}}\right), 1<p<\infty$. Theorems used here give us sufficient conditions for the existence of common solutions to the infinite family of quasi-nonexpansive multivalued operators in the uniformly convex real Banach space. The results are affected by hemicompactness, compactness, contraction properties, and the one-step iterative scheme.

It is worth remarkable to mention that the field of studying the existence and uniqueness of solutions to fractional differential equations has drawn attention of many contributors [21-28].

\section{Preliminaries}

We present this section with some needed definitions, facts, lemmas, theorems, and auxiliary results used to start the main theorems.
2.1. Real Sequence Banach Space. Define the space $\mathbb{V}$ to be the real sequence Banach space $\ell_{p}\left(\mathbb{R}^{\mathbb{N}}\right), 1<p<\infty$, endowed with the norm

$$
\begin{aligned}
\|V\|_{\ell_{p}} & =\left(\sum_{i=1}^{\infty}\left|v_{i}\right|^{p}\right)^{(1 / p)}, \\
V & =\left(v_{i}\right)_{i \in \mathbb{N} .}
\end{aligned}
$$

Then, from [29-31], we have the following facts.

Definition 1. (uniformly convex space). A normed space $\mathbb{V}$ is called a uniformly convex space if for any $\varepsilon \in(0,2]$, there exists $\delta=\delta(\varepsilon)>0$ such that if $v_{1}, v_{2} \in \mathbb{V}$ with $\left\|v_{1}\right\|=\left\|v_{2}\right\|=$ 1 and $\left\|v_{2}-v_{1}\right\| \geq \varepsilon$, then $\left\|(1 / 2)\left(v_{1}+v_{2}\right)\right\| \leq 1-\delta$.

Lemma 1. The space $\ell_{p}$ is uniformly convex for all $1 \leq p<\infty$.

Theorem 1. Adopt $1 \leq p, q<\infty$ such that $(1 / p)+(1 / q)=1$. Then, the inequalities thereafter hold:

(i) Holder inequality: let $\eta \in \ell_{p}$ and $\eta \in \in \ell_{q}$. Then, $\eta \eta \prime \in \ell_{1}$ and

$$
\|\eta \eta \prime\|_{\ell_{1}} \leq\|\eta\|_{\ell_{p}}\|\eta \prime\|_{\ell_{q}} .
$$

(ii) Minkowski inequality: let $\eta, \eta \prime \in \ell_{p}$. Then, $\eta+\eta \prime \in \ell_{p}$ and

$$
\|\eta+\eta \prime\|_{\ell_{p}} \leq\|\eta\|_{\ell_{p}}+\|\eta \prime\|_{\ell_{p}} .
$$

(iii) Imbedding theorem: let $\Omega$ have a finite positive measure and $1 \leq q \leq p \leq \infty$. Then, $\ell_{q}(\Omega) \subseteq \ell_{p}(\Omega)$ and

$$
\|\eta\|_{\ell_{p}} \leq\|\eta\|_{\ell_{q}}
$$

Theorem 2. (compactness in $\ell_{p}$ ). Let $E$ be a Banach space and $K \subset \ell_{p}(E)$. Then, $K$ is compact in $\ell_{p}(E)$ if and only if

(i) $K$ is closed and bounded

(ii) $\left(\sum_{r=n}^{\infty}\left|v_{r}\right|^{p}\right)^{(1 / p)} \longrightarrow 0$ as $n \longrightarrow \infty$

(iii) If $D_{r}: \ell_{p} \quad(E) \longrightarrow$ E defined for all $\left(v_{r}\right) \in \ell_{p}$ by $D_{r}\left[\left(v_{r}\right)_{r \geq 1}\right]=v_{r}$, then $D_{r}(K)$ is compact for all $r \geq 1$

Corollary 1. The subsets

$$
K_{x}=\left\{\left(v_{r}\right)_{r \geq 1} \in \ell_{p}|| v_{r}|\leq| x_{r}|,| x_{r} \mid \longrightarrow 0 \text { as } r \longrightarrow 0,1 \leq p \leq \infty\right\}
$$

are compact subsets of $\ell_{p}$ spaces.

2.2. Basics in Multivalued Maps. The concept of multioperators is related to the multivalued maps. So, it is required to show some facts about them and their properties. These facts are confirmed in [32-37]. 
Let $(E,\|\|$.$) and (H,\|\|$.$) be two Banach spaces. A$ multivalued map $A: E \longrightarrow P_{c l}(E)$ is seen as convex (closed) if for every $e \in E$, then $A(e)$ is convex (closed) and selected to be completely continuous if $A(B)$ is relatively compact for every $B \in P_{b}(E)$.

The map $A$ is said to be upper semicontinuous if for each closed subset $W \subset E, A^{-1}(W)$ is a closed subset of $E$. This means the set $\{e \in E: A(e) \subseteq O\}$ is open for all open sets $O \subset E$. It is lower semicontinuous if for each open subset $Z \subset E, A^{-1}(Z)$ is an open subset of $E$. In other words, $A$ seems to be lower semicontinuous as long as the set $\{e \in E: A(e) \cap O \neq \varnothing\}$ is open for all open sets $O \subset E$.

A map $A:[0, \varepsilon] \longrightarrow P_{c l}(E)$ is presented to be measurable multivalued if for every $e \in E$, the function $\tau \longrightarrow d(e, A(\tau))=\inf \{d(e, a): a \in A(\tau)\}$ is $\mathscr{L}$-measurable function.

Given $C, B \in P_{c l}(E)$; then,

$$
\begin{aligned}
h(C, B) & =H_{d}(C, B)=d_{H}(C, B) \\
& =\max \left\{\sup _{c \in C} d(c, B), \sup _{b \in B} d(C, b)\right\}
\end{aligned}
$$

absorbs the Pompeiu-Hausdorff distance of $C, B$.

If we adopt $A$ as a completely continuous function with nonempty compact values, then it is upper semicontinuous if and only if its graph is closed (i.e., if $v_{n} \longrightarrow v_{*}$ and $y_{n} \longrightarrow y_{*}$, then $y_{n} \in A\left(v_{n}\right)$ implies to $\left.y_{*} \in A\left(v_{*}\right)\right)$.

Definition 2. A multivalued map $A:[a, b] \times \mathbb{R} \longrightarrow P(\mathbb{R})$ is known as Caratheodory if

(1) $\forall r_{n} \in \mathbb{R}, n \in \mathbb{N}, \quad \tau \longrightarrow A\left(\tau,\left\{r_{n}\right\}\right)$ is measurable

(2) For a.e. $\tau \in[a, b],\left(\left\{r_{n}\right\}\right) \longrightarrow A\left(\tau,\left\{r_{n}\right\}\right)$ is upper semicontinuous

In addition to assumptions (1) and (2), the map $A$ is $L^{1}$-Caratheodory if for each $k>0, \exists \phi_{k} \in L^{1}[a, b]$ satisfying $\sup _{\tau \geq 0}\left|\phi_{k}(\tau)\right|<+\infty$ and $\phi_{k}>0$ and nondecreasing map $€$ for which

$$
\begin{aligned}
\left\|A\left(\tau,\left\{r_{n}\right\}\right)\right\| & =\sup \left\{|a|: a(\tau) \in A\left(\tau,\left\{r_{n}\right\}\right)\right\} \\
& \leq \phi_{k}(\tau) €(\|\mathrm{r}\|), \mathrm{r}=\left(\mathrm{r}_{\mathrm{n}}\right),
\end{aligned}
$$

for all $\|r\|<k, n \in \mathbb{N}, \tau \in[a, b]$.

Definition 3 (nonexpansive and quasi-nonexpansive multimapping). Let $K$ be a subset of a metric space $E$ and $T: K \longrightarrow P_{c l, b}(K) \quad$ be a multivalued map with $\operatorname{Fix}(T)=\{v \in K \mid T v=v\}$ (the set of all fixed points of $T$ in $K)$. Then, we have the following:

(i) $T$ is called a nonexpansive mapping if it is contraction according to the metric. This means that, for all $v_{1}, v_{2} \in K$, we have

$$
H_{d}\left(T v_{2}, T v_{1}\right) \leq\left\|v_{2}-v_{1}\right\|_{E}
$$

(ii) $T$ is said to be quasi-nonexpansive if $\operatorname{Fix}(T) \neq \varnothing$ and for all $w \in \operatorname{Fix}(T)$ and all $v \in K$, we have

$$
H_{d}(T v, T w) \leq\|v-w\|_{E} .
$$

(iii) $\operatorname{Fix}(T)$ is a closed subset if $K$ is closed and convex of $E$.

Definition 4 (hemicompactness). Let $K, E$, and $T$ be defined as in Definition 3. Then, $T$ is called hemicompact if

(i) For any sequence $\left(v_{r}\right)_{r \in \mathbb{N}} \in K$ such that $d\left(v_{r}\right.$, $\left.T v_{r}\right) \longrightarrow 0$ as $r \longrightarrow 0$, there is a subsequence $\left(v_{r}^{s}\right)$ of $\left(v_{r}\right)$ with $v_{r}^{s} \longrightarrow w \in K$

(ii) $T$ is compact

Theorem 3 (nonempty infinite intersection). For any space $W$, the following statements are equivalent:

(i) $W$ is compact.

(ii) Every decreasing sequence $\left\{W_{n} \mid n \in \mathbb{N}\right\}$ of nonempty closed subsets of $W$ has a nonempty intersection

$$
\text { ((i.e) } \left.W_{n+1} \subseteq W_{n}, \quad \forall n \in \mathbb{N} \Rightarrow \underset{n \geq 1}{\cap} W_{n} \neq \varnothing\right) \text {. }
$$

(iii) Every collection $\left\{W_{n} \mid n \in \mathbb{N}\right\}$ of nonempty closed subsets of $W$ satisfying the finite intersection property has a nonempty intersection

$$
\text { ((i.e) } \left.\bigcap_{s=1}^{m} W_{n_{s}} \neq \varnothing \Rightarrow \underset{n \geq 1}{\cap} W_{n} \neq \varnothing\right) \text {. }
$$

2.3. Fractional Calculus. In this part, we give definitions related to the used derivative, its history, the corresponding integral, and some properties [38-40].

Definition 5 (Riemann-Liouville integral). For the order $\zeta>0$, the Riemann-Liouville fractional integral of a function $h(\tau):[0, \infty) \longrightarrow \mathbb{R}$ is defined by

$$
I^{\zeta} h(\tau)=\frac{1}{\Gamma(\varsigma)} \int_{0}^{\tau}(\tau-\varsigma)^{\zeta-1} h(\varsigma) \mathrm{d} \varsigma
$$

since the R.H.S is pointwise defined on $(0, \infty)$.

Definition 6 (Caputo-Fabrizio derivative). CF derivative for the order $\alpha \in[0,1]$ and $\eta(\varsigma) \in H^{1}(a, b)$ is given by

$$
{ }^{\mathrm{CF}} D^{\alpha} \eta(\varsigma)=\frac{(2-\alpha) M(\alpha)}{2(1-\alpha)} \int_{a}^{\varsigma} e^{-\left(\alpha \left(\varsigma^{-s) / 1-\alpha)}\right.\right.} \eta^{\prime}(s) \mathrm{d} s,
$$

where $M(\alpha)$ is a normalization function such that $M(0)=M(1)=1$.

Definition 7 (Mittag-Leffler function). The general form of Mittag-Leffler function $E_{\alpha}$ of order $\alpha$ is written by

$$
E_{\alpha}(v)=\sum_{r=0}^{\infty} \frac{v^{r}}{\Gamma(\alpha r+1)} \text {. }
$$


The main derivative used in the present paper is the Atangana-Baleanu fractional derivative in the Caputo sense. It is proposed by interchanging the kernel $\exp (-(\alpha(\varsigma-s) / 1-\alpha))$ in the Caputo-Fabrizio derivative by the equivalent form via the Mittag-Leffler formula that is $\sum_{r=0}^{\infty}\left((-a(t-s))^{r} / r !\right), a=(\alpha / 1-\alpha)$. After that, replace $r$ ! by $\Gamma(\alpha r+1)$ and $(-a(t-s))^{r}$ by $(-a(t-s))^{\alpha r}$. So, in the space

$$
H^{1}(\Omega)=\left\{\eta(\varsigma) \mid \eta(\varsigma), D^{\rho} \eta(\varsigma) \in L^{2}(\Omega), \quad \forall \rho \leq 1\right\},
$$

we have the following definitions.

Definition 8 (Atangana-Baleanu fractional derivative in the Caputo sense). ABC derivative for the order $\alpha \in[0,1]$ and $\nu(\varsigma) \in H^{1}(a, b)$ is given by

$$
\mathrm{ABC}^{\alpha} \nu(\varsigma)=\frac{M(\alpha)}{(1-\alpha)} \int_{a}^{\varsigma} E_{\alpha}\left[-\frac{\alpha(\varsigma-s)^{\alpha}}{1-\alpha}\right] \nu /(s) \mathrm{d} s .
$$

This derivative is related to the fading memory concept and frequently used to discuss and analyze the real-world phenomena such as fluid and nanofluid models (see [41-43] and references therein). Depending on the constant $M(\alpha)$, the corresponding integral is given by the following definition.

Definition 9 (Atangana-Baleanu fractional integral). AB integral of the order $\alpha \in[0,1]$ and $\nu(\varsigma) \in H^{1}(a, b)$ is given by

$$
{ }_{\mathrm{AB}} I^{\alpha} \nu(t)=\frac{1-\alpha}{M(\alpha)} \nu(t)+\frac{\alpha}{M(\alpha)} R_{a}^{\alpha} \nu(t)
$$

where ${ }_{R} I^{\alpha}$ is the Riemann-Liouville integral of order $\alpha$.

Lemma 2. For $\alpha \in(0,1), t \in(a, b)$, and $v(t) \in A C^{1}[a, b]$, the following statements hold:

a1: $A B$ integral together with the $A B C$ derivative satisfies the Newton-Leibniz formula

$$
{ }_{\mathrm{AB}} I_{a^{+}}^{\alpha}\left[{ }_{\mathrm{ABC}} D_{a^{+}}^{\alpha} \nu\right](t)=v(t)-v(a) .
$$

a2: they also satisfy the property

$$
{ }_{\mathrm{ABC}} D_{0^{+}}^{\alpha}\left[{ }_{\mathrm{AB}} I_{0^{+}}^{\alpha} \nu\right](t)=\nu(t)-\nu(o) E_{\alpha}\left(\frac{-\alpha t^{\alpha}}{1-\alpha}\right) .
$$

a3: AB integral is a commutative operator such that, for any two orders $\alpha, \beta \in(0,1)$, we have

$$
\begin{aligned}
\left.\mathrm{AB}_{a^{+}} I_{\mathrm{AB}}^{\alpha} I_{a^{+}}^{\beta} \nu\right](t) & =\left[\frac{1-\alpha}{M(\alpha)}+\frac{\alpha}{M(\alpha)}{ }_{\mathrm{R}} I_{a}^{\alpha}\right]\left[\frac{1-\beta}{M(\beta)}+\frac{\beta}{M(\beta)}{ }_{\mathrm{R}} I_{a}^{\beta}\right] \\
\nu(t) & =\left[\frac{1-\beta}{M(\beta)}+\frac{\beta}{M(\beta)}{ }_{\mathrm{R}} I_{a}^{\beta}\right]\left[\frac{1-\alpha}{M(\alpha)}+\frac{\alpha}{M(\alpha)}{ }_{\mathrm{R}} I_{a}^{\alpha}\right] \\
\nu(t) & ={ }_{\mathrm{AB}} I_{a^{+}}^{\beta}\left[{ }_{\mathrm{AB}} I_{a^{+}}^{\alpha} \nu\right](t) .
\end{aligned}
$$

Lemma 3 (antiperiodic solution). The unique solution of the problem

$$
\begin{gathered}
\operatorname{ABC}^{\alpha}[\nu(t)]=\varepsilon(t), \quad t \in[0, \rho], \\
\sigma \nu(0)=\gamma \nu(\rho), \sigma, \quad \gamma \in \mathbb{R}, \text { with } \sigma \neq \gamma, \alpha \in[0,1],
\end{gathered}
$$

is given by

$$
\begin{aligned}
\nu(t)= & \frac{1-\alpha}{M(\alpha)} \varepsilon(t)+\frac{\alpha}{M(\alpha) \Gamma(\alpha)} \int_{0}^{t}(t-s)^{\alpha-1} \varepsilon(s) \mathrm{d} s \\
& +\frac{\gamma}{\sigma-\gamma}\left[\frac{1-\alpha}{M(\alpha)}\left(\varepsilon(\rho)-\frac{\sigma}{\gamma} \varepsilon(0)\right)\right. \\
& \left.+\frac{\alpha}{M(\alpha) \Gamma(\alpha)} \int_{0}^{\rho}(\rho-s)^{\alpha-1} \varepsilon(s) \mathrm{d} s\right] .
\end{aligned}
$$

Proof. By applying ${ }_{\mathrm{AB}} I^{\alpha}$ to both sides of (23), we get

$$
\nu(t)={ }_{\mathrm{AB}} I^{\alpha}(\varepsilon)(t)+c
$$

which implies

$$
\begin{aligned}
& \nu(0)=\frac{1-\alpha}{M(\alpha)} \varepsilon(0)+c, \\
& \nu(\rho)={ }_{\mathrm{AB}} I^{\alpha}(\varepsilon)(\rho)+c .
\end{aligned}
$$

Now, applying (24), we find that

$$
\begin{aligned}
c= & \frac{\gamma}{\sigma-\gamma}\left[\frac{1-\alpha}{M(\alpha)}\left(\varepsilon(\rho)-\frac{\sigma}{\gamma} \varepsilon(0)\right)\right. \\
& \left.+\frac{\alpha}{M(\alpha) \Gamma(\alpha)} \int_{0}^{\rho}(\rho-s)^{\alpha-1} \varepsilon(s) \mathrm{d} s\right] .
\end{aligned}
$$

Lemma 4 (periodic/almost periodic solution). The unique solution of the problem

$$
\begin{aligned}
\mathrm{ABC}^{\alpha} D^{\alpha}[(t)] & =\varepsilon(t), \quad t \in[0, \rho], \\
\nu(0) & \approx \nu(\rho), \quad \alpha \in[0,1],
\end{aligned}
$$

is given by

$$
\begin{gathered}
\nu(t)={ }_{\mathrm{AB}} I^{\alpha}(\varepsilon)(t)+c \text { whenever } \\
\varepsilon(0) \approx \varepsilon(\rho),
\end{gathered}
$$

$$
\int_{0}^{\rho} \frac{(\rho-s)^{\alpha-1}}{\Gamma(\alpha)} \varepsilon(s) \mathrm{d} s \approx 0,
$$

and then $c \approx-(1-\alpha / M(\alpha)) \mathcal{\varepsilon}(0) \approx-(1-\alpha / M(\alpha)) \mathcal{\varepsilon}(\rho)$.

Proof. Similar to the proof of Lemma 3, we have

$$
\nu(t)={ }_{\mathrm{AB}} I^{\alpha}(\varepsilon)(t)+c .
$$

The almost periodic/periodic condition leads to 


$$
(1-\alpha)(\varepsilon(\rho)-\varepsilon(0))+\frac{\alpha}{\Gamma(\alpha)} \int_{0}^{\rho}(\rho-s)^{\alpha-1} \varepsilon(s) \mathrm{d} s \simeq 0 .
$$

Under this equation, we have three cases:

p1: if $\alpha=0$, then $\varepsilon(\rho) \approx \varepsilon(0)$ must hold

p2: when $\quad \alpha \in(0,1)$, both $\quad \varepsilon(\rho) \approx \varepsilon(0)$ and $\int_{0}^{\rho}\left((\rho-s)^{\alpha-1} / \Gamma(\alpha)\right) \varepsilon(s) \mathrm{d} s \approx 0$ must hold

p3: in case $\alpha=1$, we get $\int_{0}^{\rho} \varepsilon(s) \mathrm{d} s \approx 0$

So, we can say, in general, that there is an almost periodic/periodic solution if both (31) and (32) hold for every $\alpha \in[0,1]$. Now, substituting (31) and (32) to the value of $c=c(\sigma)$ in Lemma 3 with $\sigma \longrightarrow \gamma$ and using the continuity condition, we get $\lim _{\sigma \longrightarrow \gamma} c=-((1-\alpha / M(\alpha))) \varepsilon(0)=\left.c\right|_{\sigma=\gamma}$. Hence, the periodic/almost periodic solution is given by

$$
\begin{aligned}
v(t) & ={ }_{\mathrm{AB}} I^{\alpha}(\varepsilon)(t)-\left(\frac{1-\alpha}{M(\alpha)}\right) \varepsilon(0) \\
& =\frac{1-\alpha}{M(\alpha)}[\varepsilon(t)-\varepsilon(0)]+\frac{\alpha}{M(\alpha) \Gamma(\alpha)} \int_{0}^{t}(t-s)^{\alpha-1} \varepsilon(s) \mathrm{d} s .
\end{aligned}
$$

It is clear to see that $\nu(0) \cong 0 \cong \nu(\rho)$ which completes the proof.

2.4. Fixed-Point Theorems. This section is surveyed by some fixed-point theorems investigated in the uniformly convex real Banach space [44, 45].

Theorem 4. Let $\mathbb{V}$ be a uniformly convex real Banach space and $K$ be a closed, bounded, convex subset of $\mathbb{V}$. Let $T: K \longrightarrow P_{c p}(K)$ be nonexpansive multivalued mappings. Then, $T$ has a fixed point $x \in K$ with $x \in T x$.

The next theorem is formulated for the infinite countable family of multioperators under the vision of the one-step iterative scheme defined as follows.

Let $K$ be a closed, bounded, convex subset of a uniformly convex real Banach space $\mathbb{V}$. Let $\left\{T_{i}: K \longrightarrow P_{b}(K)\right\}$ be an infinite countable family of quasi-nonexpansive multivalued mappings with $\cap_{i=1}^{\infty}$ Fix $\left(T_{i}\right) \neq \varnothing$ and $p \in \cap_{i=1}^{\infty} \operatorname{Fix}\left(T_{i}\right)$. Then, for all $n \in \mathbb{N}$, the sequence $\left\{V_{n}\right\}$ is defined by

$$
\begin{gathered}
V_{1} \in K, \quad V_{n+1}=\sigma_{n, 0} V_{n}+\sum_{r=1}^{n} \sigma_{n, r} V_{n, r}, \\
V_{n, r} \in T_{r} V_{n} \text {, such that }\left\|p-V_{n, r}\right\|=d\left(p, T_{r} V_{n}\right), \\
\left\{\sigma_{n, r}\right\} \subset[0,1) \text {, with } \sum_{r=0}^{n} \sigma_{n, r}=1, \quad r \in \mathbb{N} .
\end{gathered}
$$

Theorem 5. Let $K$ be a closed, bounded, convex subset of a uniformly convex real Banach space $\mathbb{V}$. Let $\left\{T_{i}: K \longrightarrow P_{b}(K)\right\}$ be a sequence of quasi-nonexpansive and continuous multivalued mappings with $\cap_{i=1}^{\infty} \operatorname{Fix}\left(T_{i}\right) \neq \varnothing$ and $p \in \cap_{i=1}^{\infty}$ Fix $\left(T_{i}\right)$. Let $\left\{V_{i}\right\}$ be a sequence defined by (36) with the condition that $\lim _{n \longrightarrow \infty} \sigma_{n, r}$ and $\lim _{n \longrightarrow \infty} \sigma_{n, n}$ exist and lie in $[0,1)$ for all $r \in\{0\} \cup \mathbb{N}$. Assume that one of $\left\{T_{i}\right\}$ is hemicompact. Then, the sequence $\left\{V_{i}\right\}$ converges strongly.

\section{Inferred Results}

3.1. Auxiliary Results. Let $V(t): C[0, \rho] \longrightarrow \mathbb{R}^{\mathbb{N}}$ be defined by $V(t)=\left(v_{i}(t)\right)_{i \in \mathbb{N}}$. Then, for every multivalued mapping $y_{i}$, we have $y_{i}\left(t, v_{1}(t), \ldots, v_{i}(t), \ldots\right)=y_{i}(t, V(t))$. Define the set-valued maps $S_{\mathscr{Y}_{i}, V}$ such as

$$
S_{\mathscr{Y}_{i}, V}=\left\{\varepsilon_{i}(t) \mid \varepsilon_{i}(t) \in \mathscr{y}_{i}(t, V(t)) \cap L^{1}(J, \mathbb{R})\right\}, \quad J=[0, \rho] .
$$

For the antiperiodic solutions, we define the multioperators $\left\{\Upsilon_{i}: \ell_{p} \longrightarrow \mathbb{R}\right\}$ for all $i \in \mathbb{N}$ as follows:

$$
\left.\left(\Upsilon_{i} V\right)(t)=\left\{\Psi_{\mathscr{y}_{i}}(t) \mid \Psi_{\mathscr{y}_{i}}(t)=\Delta\left(\varepsilon_{i}\right)(t), \quad \varepsilon_{i}(t)\right) \in \overline{S_{\mathscr{Y}_{i}, V}}\right\},
$$

where

$$
\begin{aligned}
\Delta\left(\varepsilon_{i}\right)(t)= & \frac{1-\alpha}{M(\alpha)} \varepsilon_{i}(t)+\frac{\alpha}{M(\alpha) \Gamma(\alpha)} \int_{0}^{t}(t-s)^{\alpha-1} \varepsilon_{i}(s) \mathrm{d} s \\
& +\frac{\gamma}{\sigma-\gamma}\left[\frac{1-\alpha}{M(\alpha)}\left(\varepsilon_{i}(\rho)-\frac{\sigma}{\gamma} \varepsilon_{i}(0)\right)\right. \\
& \left.+\frac{\alpha}{M(\alpha) \Gamma(\alpha)} \int_{0}^{\rho}(\rho-s)^{\alpha-1} \varepsilon_{i}(s) \mathrm{d} s\right] .
\end{aligned}
$$

For the almost periodic/periodic solutions, the multioperators $\left\{\Pi_{i}: \ell_{p} \longrightarrow \mathbb{R}\right\}$ for all $i \in \mathbb{N}$ are defined by

$$
\left(\Pi_{i} V\right)(t)=\left\{\Theta_{\mathscr{y}_{i}}(t) \mid \Theta_{\mathscr{y}_{i}}(t)=\underline{\Delta}\left(\varepsilon_{i}\right)(t), \varepsilon_{i}(t) \in \overline{S_{\mathscr{y}_{i}, V}}\right\},
$$

where

$$
\begin{aligned}
\underline{\Delta}\left(\varepsilon_{i}\right)(t)= & \frac{1-\alpha}{M(\alpha)}\left[\varepsilon_{i}(t)-\varepsilon_{i}(0)\right] \\
& +\frac{\alpha}{M(\alpha) \Gamma(\alpha)} \int_{0}^{t}(t-s)^{\alpha-1} \varepsilon_{i}(s) \mathrm{d} s .
\end{aligned}
$$

Consider that the following conditions hold:

$\left(\mathrm{H}_{1}\right)\left(y_{i}\right)$ is a sequence of $L^{1}$-Caratheodory multivalued mappings that are

$$
y_{i}: J \times \mathbb{V} \longrightarrow P_{c p}(\mathbb{R}),
$$

with

(1) The maps $t \longrightarrow y_{i}(t, V(t))$ are measurable for all $V \in \mathbb{V}$.

(2) The maps $V \longrightarrow y_{i}(t, V(t))$ are measurable for all $t \in J$.

(3) For all $i \in \mathbb{N}$, there exist $\psi_{i} \in C\left[J, \mathbb{R}_{+}\right]$such that

$\left(\mathrm{H}_{2}\right) \mathrm{y}_{i}$ are Lipschitz mappings with constants $L_{i} \in \mathbb{R}_{+}$, respectively. This means 
$H_{d}\left(\mathscr{Y}_{i}\left(t, V_{1}(t)\right), \mathscr{Y}_{i}\left(t, V_{2}(t)\right)\right) \leq L_{i}\left\|V_{1}-V_{2}\right\|, \quad \forall i \in \mathbb{N}$.

$\left(\mathscr{H}_{3}\right) \sum_{i \geq 1} L_{i}<\infty$ and $Z=\Xi \sum_{i=1}^{\infty} L_{i}<1$ whenever

$$
\Xi=2\left(\frac{1-\alpha}{M(\alpha)}\right)+\left(1+\left|\frac{\gamma}{\sigma-\gamma}\right|\right) \frac{\rho^{\alpha}}{M(\alpha) \Gamma(\alpha)} .
$$

$\left(\mathscr{H}_{4}\right)\left(\left|v_{i}\right| / \Xi \psi_{i}^{*}\left|v_{i}\right|\right)>1, \psi_{i}^{*}=\sup _{t \in J}\left|\psi_{i}(t)\right|$.

Then, we have the following propositions.

Proposition 1. The set-valued maps $\Upsilon_{i}(V)$ are bounded and contraction for all $i \in \mathbb{N}$.

Proof. In view of $\left(\mathscr{H}_{1}\right)$ and $\left(\mathscr{H}_{4}\right)$, we have

$$
\begin{aligned}
\left|\Upsilon_{i}(V)\right| \leq & {\left[\frac{1-\alpha}{M(\alpha)}+\frac{t^{\alpha}}{M(\alpha) \Gamma(\alpha)}+\left|\frac{\gamma}{\sigma-\gamma}\right|\left(\left|1-\frac{\sigma}{\gamma}\right|\left(\frac{1-\alpha}{M(\alpha)}\right)\right.\right.} \\
& \left.\left.+\frac{\rho^{\alpha}}{M(\alpha) \Gamma(\alpha)}\right)\right]\left|\mathscr{Y}_{i}(t, V(t))\right| \\
\leq & \Xi \psi_{i}^{*}\left|v_{i}\right| \leq\left|v_{i}\right| .
\end{aligned}
$$

Thus, we prove the boundedness.

To prove the contraction condition for all $i \in \mathbb{N}$, consider that $\Psi_{\mathscr{Y}_{i}}^{1}(t) \in \Upsilon_{i}\left(V_{2}\right)$. This implies the existence of some $\varepsilon_{i}^{1}(t) \in \overline{S_{y_{i}, V_{2}}}$ subject to

$$
\Psi_{\mathscr{Y}_{i}}^{1}(t)=\Delta\left(\varepsilon_{i}^{1}(t)\right)
$$

where $\Delta$ is defined by $(41)$. By using $\left(\mathscr{H}_{2}\right)$, we can define the sets

$$
\bar{\Upsilon}_{i}(t)=\left\{\varepsilon \in \mathbb{R}|| \varepsilon_{i}^{1}(t)-\varepsilon(t) \mid \leq L_{i}\left\|V_{1}-V_{2}\right\|_{\ell_{p}}\right\} .
$$

According to Theorem III.41 in [46], $\left(\mathscr{H}_{1}\right)$, and the measurability of the functions $\varepsilon_{i}^{1}(t)$ and $W(t)=L_{i}\left\|V_{1}-V_{2}\right\|_{\ell_{p}}$, the sets $\bar{\Upsilon}_{i}(t)$ are also measurable for all $i \in \mathbb{N}$. Therefore, the maps $t \longrightarrow \bar{\Upsilon}_{i}(t) \cap \mathscr{Y}_{i}(t, V(t))$ are measurable with nonempty closed values. Hence, the measurability of $\Upsilon_{i}(t)$ and Proposition 2.1.43 in [47] drive to the existence of some $\varepsilon_{i}^{2} \in \overline{S_{\mathscr{y}_{i}, V_{1}}}$ for which

$$
\left|\varepsilon_{i}^{1}(t)-\varepsilon_{i}^{2}(t)\right| \leq L_{i}\left\|V_{1}-V_{2}\right\|_{\ell_{p}}, \quad i \in \mathbb{N}
$$

Define $\Psi_{\mathscr{Y}_{i}}^{2}$ by

$$
\Psi_{y_{i}}^{2}(t)=\Delta\left(\varepsilon_{i}^{2}(t)\right)
$$

which leads to

$$
\begin{aligned}
\left|\Psi_{\mathscr{y}_{i}}^{1}(t)-\Psi_{\mathscr{y}_{i}}^{2}(t)\right| & =\left|\Delta\left(\varepsilon_{i}^{1}\right)(t)-\Delta\left(\varepsilon_{i}^{2}\right)(t)\right| \\
& \leq \Xi L_{i}\left\|V_{1}-V_{2}\right\|_{\ell_{p}}, \quad i \in \mathbb{N}
\end{aligned}
$$

where $\Xi L_{i}<1$ by $\left(\mathscr{H}_{3}\right)$. Using the Akin relation obtained by interchanging the rules of $V_{1}$ and $V_{2}$, we conclude that, for all $i \in \mathbb{N}$, the operators $Y_{i}$ are contraction.

Proposition 2. For all $n \in \mathbb{N}$, define the operators $T_{n}: K_{x} \rightarrow P\left(K_{x}\right)$,

$$
\begin{aligned}
T_{n}(V) & =\left(\nu_{1}, \ldots, v_{n-1}, v_{n}, \Upsilon_{n+1}(V), \ldots\right) \\
& =\left(\left\{\begin{array}{cc}
v_{i}, & i \leq n \\
\Upsilon_{i}(V), & i>n
\end{array}\right\}\right),
\end{aligned}
$$

where $K_{x}$ is created as the one in Corollary 1. The following statements are all satisfied:

(i) $T_{n}(V) \in P_{b}\left(K_{x}\right)$ for all $n \in \mathbb{N}$

(ii) $T_{n}$ are contraction with constants $Z=\Xi \sum_{i=1}^{\infty} L_{i}$ for all $n \in \mathbb{N}$ if and only if there exist some $\Psi_{y_{i}}(t) \in \Upsilon_{i}(V)$ such that $v_{i}=\Psi_{y_{i}}(t)$

(iii) $\left\{T_{n}\right\}_{n \in \mathbb{N}}$ is a decreasing nested sequence which means $T_{n+1} \subseteq T_{n}$ for all $n \in \mathbb{N}$

Proof

(i) Using Proposition 1 and the definition of $K_{x}$, we get, for all $i \in \mathbb{N}$, that $\left|Y_{i}(V)\right| \leq\left|v_{i}\right| \leq\left|x_{i}\right|$. This implies $\left(\Upsilon_{i}(V)\right)_{i \geq 1} \in K_{x}$. Consequently, $T_{1}(V) \in P_{b}\left(K_{x}\right)$, and thus, all $T_{n}(V) \in P_{b}\left(K_{x}\right)$ for all $i \in \mathbb{N}$.

(ii) Define the metric map $G_{d}$ as

$G_{d}\left(T_{n}\left(V_{1}\right), T_{n}\left(V_{2}\right)\right)=\sum_{i=1}^{\infty} H_{d}\left(\Upsilon_{i}\left(V_{1}\right), \Upsilon_{i}\left(V_{2}\right)\right)$.

Consider that there exist some $\Psi_{y_{i}}(t) \in \Upsilon_{i}(V)$ such that $v_{i}=\Psi_{y_{i}}(t), i=1, \ldots, n$. Using the contraction result in Proposition 1, we find

$$
\begin{aligned}
G_{d}\left(T_{n}\left(V_{1}\right), T_{n}\left(V_{2}\right)\right) & \leq\left[\Xi \sum_{i=1}^{\infty} L_{i}\right]\left\|V_{1}-V_{2}\right\|_{\ell_{p}} \\
& =Z\left\|V_{1}-V_{2}\right\|_{\ell_{p}} .
\end{aligned}
$$

Applying $\left(\mathscr{H}_{3}\right)$, we get the result of the contraction condition for $T_{n}$ for all $n \in \mathbb{N}$.

Now, consider that, for all $n \in \mathbb{N}, T_{n}$ are contraction and $v_{i} \notin \Upsilon_{i}(V)$. Define the metric

$$
\begin{aligned}
\bar{G}_{d}\left(T_{n}\left(V_{1}\right), T_{n}\left(V_{2}\right)\right)= & \sum_{r=1}^{n}\left|v_{1}^{r}-v_{2}^{r}\right| \\
& +\sum_{r=n+1}^{\infty} H_{d}\left(\Upsilon_{r}\left(V_{1}\right), \Upsilon_{r}\left(V_{2}\right)\right) .
\end{aligned}
$$




$$
\begin{aligned}
& \bar{G}_{d}\left(T_{n}\left(V_{1}\right), T_{n}\left(V_{2}\right)\right) \\
& \quad \leq n\left\|V_{1}-V_{2}\right\|_{\ell_{p}}+\left[\Xi \sum_{r=n+1}^{\infty} L_{i}\right]\left\|V_{1}-V_{2}\right\|_{\ell_{p}} \\
& \quad=(n+Z)\left\|V_{1}-V_{2}\right\|_{\ell_{p}},
\end{aligned}
$$

where $(n+Z)>1$. This contradicts with the assumption that $T_{n}$ are contraction operators. Hence, we get $v_{i} \in \Upsilon_{i}(V)$.

(iii) By (ii), we have for all $n \in \mathbb{N}$ that

$$
\begin{aligned}
T_{n}(V) & =\left(\left\{\begin{array}{ll}
v_{i} & i \leq n \\
\Upsilon_{i}(V) & i>n
\end{array}\right)\right. \\
& =\left(\{ \begin{array} { l l } 
{ \Psi _ { \mathscr { Y } _ { i } } ( t ) } & { i \leq n } \\
{ \Upsilon _ { i } ( V ) } & { i > n }
\end{array} ) \supseteq \left(\left\{\begin{array}{ll}
\Psi_{\mathscr{Y}_{i}}(t) & i \leq n+1 \\
\Upsilon_{i}(V) & i>n+1
\end{array}\right)\right.\right. \\
& =\left(\begin{array}{ll}
v_{i} & i \leq n+1 \\
\Upsilon_{i}(V) & i>n+1
\end{array}\right)=T_{n+1}(V) .
\end{aligned}
$$

Proposition 3. For all $n \in \mathbb{N}$, define $T_{n}$ such as in Proposition 2. Then,

a1: for all $n \in \mathbb{N}, T_{n}$ are quasi-nonexpansive mappings

a2: for all $n \in \mathbb{N}$, Fix $\left(T_{n}\right)$ are closed subsets of $K_{x}$

a3: for all $n \in \mathbb{N}, T_{n}$ are all hemicompact mappings

a4: $\cap_{i=1}^{\infty} F i x\left(T_{n}\right) \neq \varnothing$

\section{Proof}

a1: following the theorem saying that the continuous image of the compact set is compact itself with applying Proposition 2 (I, II) implies $T_{n}\left(K_{x}\right) \subset P_{c p}\left(K_{x}\right)$. By Theorem 6 and Definition 3, we get $\operatorname{Fix}\left(T_{n}\right) \neq \varnothing$ for all $n \in \mathbb{N}$. Thus, $T_{n}$ are all quasi-nonexpansive operators. a2: from (a1) and since $K_{x}$ is a closed and convex subset of Banach space, we see that Fix $\left(T_{n}\right)$ are closed subsets of $K_{x}$ according to Definition 3.

a3: due to Definition 4 and the proof of (a1), $T_{n}$ are hemicompact for all $n \in \mathbb{N}$.

a4: let $p \in \operatorname{Fix}\left(T_{n+1}\right)$; then, $p \in T_{n+1}(p) \subseteq T_{n}(p)$. Hence, $p \in T_{n}(p)$ which follows $\operatorname{Fix}\left(T_{n+1}\right) \subseteq \operatorname{Fix}\left(T_{n}\right)$. Using Theorem 3, we get $\cap_{i=1}^{\infty} \operatorname{Fix}\left(T_{n}\right) \neq \varnothing$.

\subsection{Main Results}

Theorem 6. Consider that $\mathscr{Y}_{i}: J \times \mathbb{V} \longrightarrow P_{c p}(\mathbb{R})$ satisfy $\left(\mathscr{H}_{1}\right),\left(\mathscr{H}_{2}\right),\left(\mathscr{H}_{3}\right)$, and $\left(\mathscr{H}_{4}\right)$. Assume that, for all $n \in \mathbb{N}, T_{n}$ are defined by (40), (41), and (54). Also, let

$$
\Xi=2\left(\frac{1-\alpha}{M(\alpha)}\right)+\left(1+\left|\frac{\gamma}{\sigma-\gamma}\right|\right) \frac{\rho^{\alpha}}{M(\alpha) \Gamma(\alpha)} .
$$

Then, infinite systems (1) and (2) are able to have a common antiperiodic solution if and only if there exist some $\Psi_{y_{i}}(t) \in \Upsilon_{i}(V)$ such that $v_{i}=\Psi_{\mathscr{y}_{i}}(t)$.

Proof. Define the sequence $\left\{V_{n}\right\}_{n \geq 1}$ by (36)-(38). Under the vision of Theorem 5, Propositions 1-3 explain the existence of the common solution to infinite systems (1) and (2).

Theorem 7. Consider that $\mathscr{Y}_{i}: J \times \mathbb{V} \longrightarrow P_{c p}(\mathbb{R})$ satisfy $\left(\mathscr{H}_{1}\right),\left(\mathscr{H}_{2}\right)$, and $\left(\mathscr{H}_{4}\right)$. Assume that, for all $n \in \mathbb{N}, \bar{T}_{n}$ are defined by the same way as (54) with respect to (42) and (43). Moreover, let

$$
\bar{\Xi}=2\left(\frac{1-\alpha}{M(\alpha)}\right)+\frac{\rho^{\alpha}}{M(\alpha) \Gamma(\alpha)}
$$

and

$$
\left(\mathscr{H}_{5}\right) \sum_{i \geq 1} L_{i}<\infty \text { and } \bar{Z}=\bar{\Xi} \sum_{i=1}^{\infty} L_{i}<1 \text {. }
$$

Then, infinite systems (1) and (2) are able to have at least a common periodic solution if and only if there exist some $\Theta_{\mathscr{y}_{i}}(t) \in \Pi_{i}(V)$ such that $v_{i}=\Theta_{\mathscr{y}_{i}}(t)$.

Proof. Similar to the proof of Theorem 6 but with respect to $\bar{\Xi}$.

Corollary 2. Infinite systems (1) and (2) are able to have one or more common almost periodic solutions.

\section{Examples}

Example 1. Consider the problem

$$
\begin{gathered}
\mathrm{ABC}^{(2 / 3)}\left[v_{n}\right] \in\left[\frac{1}{2^{n r}}\left(\frac{v_{n}}{1+\sum_{s=1}^{r}\left|v_{s}\right|}\right)\right]_{r=1}^{n}, \\
3 V(0)=V(\rho) .
\end{gathered}
$$

Then, we have $\mathscr{Y}_{n}(t, V(t))=\left[\left(1 / 2^{n r}\right) \quad\left(v_{n} / 1+\right.\right.$ $\left.\left.\sum_{s=1}^{r}\left|v_{s}\right|\right)\right]_{r=1}^{n}$ and

$$
\left|\mathscr{Y}_{n}\right| \leq \frac{1}{2^{n}}\left|v_{n}\right| .
$$

If we take $\psi_{n}(t)=\left(1 / 2^{n}\right)=\psi_{n}^{*}$, we find that $\left(\mathscr{H}_{1}\right)$ holds. Furthermore, we have

$$
\begin{gathered}
H_{d}\left(\mathscr{Y}_{n}\left(t, V_{1}(t)\right), \mathscr{Y}_{n}\left(t, V_{2}(t)\right)\right) \leq \frac{1}{2^{n}}\left|v_{1}^{n}-v_{2}^{n}\right| \\
=\frac{1}{2^{n}}\left(\left|v_{1}^{n}-v_{2}^{n}\right|^{P}\right)^{(1 / p)} \leq \frac{1}{2^{n}}\left\|V_{1}-V_{2}\right\|_{\ell_{p}}
\end{gathered}
$$

which explains that $\left(\mathscr{H}_{2}\right)$ holds with $L_{n}=\left(1 / 2^{n}\right)$. It is known that $\sum_{n=1}^{\infty} L_{n}=\sum_{n=1}^{\infty}\left(1 / 2^{n}\right)=1<\infty$. In this case, we need to make sure that $\Xi<1$ for the sake of obtaining $\left(\mathscr{H}_{3}\right)$. First, by using the rules 


$$
\begin{aligned}
M(\alpha) & =\frac{2}{2-\alpha}, \\
\Gamma(\alpha) \Gamma(1-\alpha) & =\frac{\pi}{\sin \alpha \pi},
\end{aligned}
$$

we find that

$$
\begin{aligned}
M\left(\frac{2}{3}\right) & =\frac{3}{2}, \\
\Gamma\left(\frac{1}{3}\right) & \approx 2.6789385347077476337, \\
\frac{1}{M(2 / 3) \Gamma(2 / 3)} & \approx 0.0086, \\
\left(1+\left|\frac{\gamma}{\sigma-\gamma}\right|\right) \frac{\rho^{\alpha}}{M(\alpha) \Gamma(\alpha)} & \approx 0.0129 \rho^{(2 / 3)}, \\
2\left(\frac{1-\alpha}{M(\alpha)}\right) & =\frac{4}{9} .
\end{aligned}
$$

Taking $\quad \rho^{(2 / 3)} \leq(2 / 9(0.0129)) \quad$ implies that $\rho \in(0,10.5881117)$. Therefore, $\Xi \leq(8 / 9)<1$ which means that $\left(\mathscr{H}_{3}\right)$ holds. Finally, since $\psi_{n}^{*} \Xi \leq\left(8 / 9\left(2^{n}\right)\right)<1, n \geq 1$, $\left(\mathscr{H}_{4}\right)$ holds. Applying Theorem 6, there exists at least one solution of antiperiodic type for problems (62) and (63).

Example 2. Assume the following problem:

$$
\begin{aligned}
\mathrm{ABC}^{1}\left[\nu_{n}\right] & \in\left[\frac{1}{(2+r)^{n}}\left(\frac{\sin \nu_{n}}{1+\sum_{s=1}^{r}\left|\nu_{s}\right|}\right)\right]_{r=1}^{n}, \\
V(0) & =V(\rho) .
\end{aligned}
$$

In this problem, we have $\alpha=M(\alpha)=1$,

$$
\begin{aligned}
\mathscr{Y}_{n}(t, V(t))= & {\left[\frac{1}{(2+r)^{n}}\left(\frac{\sin v_{n}}{1+\sum_{s=1}^{r}\left|v_{s}\right|}\right)\right]_{r=1}^{n}, } \\
& \left|\mathscr{Y}_{n}\right| \leq \frac{1}{3^{n}}\left|\nu_{n}\right| .
\end{aligned}
$$

It follows that $\psi_{n}(t)=\left(1 / 3^{n}\right)=\psi_{n}^{*}$, and thus, $\left(\mathscr{H}_{1}\right)$ holds. It is clear to see that $\left(\mathscr{H}_{2}\right)$ holds if we take $L_{n}=\left(1 / 3^{n}\right)$ and then $\sum_{n=1}^{\infty} L_{n}=(1 / 2)<1$. Since $\alpha=1, \bar{\Xi}=\rho$, and $\bar{Z}=$ $(\rho / 2)$ tends to take $\rho \in(0,2)$ in order to obtain $\left(\mathscr{H}_{5}\right)$. Finally, we have $\left(1 / \Xi \psi_{n}^{*}\right)=\left(3^{n} / \rho\right)>(3 / 2)>1$ which drives to $\left(\mathscr{H}_{6}\right)$. According to Theorem 7, problems (42), (43), (54), (62), (63), (68), and (69) have at least one periodic solution.

Remark 1. If we take problems (42), (43), (54), (62), (63), (68), and (69) with the condition $V(0) \approx V(\rho)$, then by the same results in Example 2, we get the existence result of the almost periodic solution.

\section{Conclusion}

By this work, we connected between three sides of generalization: first, using the generalization of fractional differential operators with the Mittag-Leffler kernel; second, generating the infinite countable system of equations by multivalued mappings; third, using the general form of periodic, almost periodic, and antiperiodic boundary conditions. We explain how to obtain the exact solutions in the three cases according to the boundary conditions. Consequently, we show the sufficient conditions for the existence results to different solutions and give some related examples to the main theorems. These results have strong impacts to give an even better description of the dynamics of real-world problems (in particular, the dynamics complex systems). These results also have practical extensions to understand the complex phenomena related to multifaceted media, chaotic behaviors, fluctuations, nanofluids, and heterogeneous phenomenon. Next time, we will work to study the generalization of the conjugate value problem with one of the most important derivatives.

\section{Data Availability}

The data used to support the findings of this study are included within the article, and other data used can be obtained from the corresponding author upon request.

\section{Conflicts of Interest}

The authors declare that they have no conflicts of interest.

\section{Authors' Contributions}

Both authors contributed equally to this work and read and approved the final manuscript.

\section{Acknowledgments}

This work was supported by the Deanship of Scientific Research (DSR), King Abdulaziz University, Jeddah (Grant no. D-146-130-1439). The authors, therefore, gratefully acknowledge the DSR for the technical and financial support.

\section{References}

[1] J. Banas and M. Lecko, "Solvability of infinite systems of differential equations in Banach sequence spaces," Journal of Computational and Applied Mathematics, vol. 137, pp. 363375, 2001.

[2] M. Mursaleen and S. A. Mohiuddine, "Applications of measures of noncompactness to the infinite system of differential equations in $\ell_{p}$ spaces," Nonlinear Analysis: Theory, Methods \& Applications, vol. 75, no. 4, pp. 2111-2115, 2012.

[3] M. Mursaleen, "Application of measure of noncompactness to infinite systems of differential equations," Canadian Mathematical Bulletin, vol. 56, no. 2, pp. 388-394, 2013.

[4] S. A. Mohiuddine, H. M. Srivastava, and A. Alotaibi, "Application of measures of noncompactness to the infinite 
system of second-order differential equations in $\ell_{p}$ spaces," Advances in Difference Equations, vol. 2016, pp. 1-13, 2016.

[5] M. Mursaleen, B. Bilalov, and S. Rizvi, "Applications of measures of noncompactness to infinite system of fractional differential equations," Filomat, vol. 31, no. 11, pp. 3421-3432, 2017.

[6] F. Wang and Y. Cui, "Positive solutions for an infinite system of fractional order boundary value problems," Advances in Difference Equations, vol. 169, 2019.

[7] F. Wang, Y. Cui, and H. Zhou, "Solvability for an infinite system of fractional order boundary value problems," Annals of Functional Analysis, vol. 10, no. 3, pp. 395-411, 2019.

[8] A. Seemab and M. U. Rehman, "Existence of solution of an infinite system of generalized fractional differential equations by darbo's fixed point theorem," Journal of Computational and Applied Mathematics, vol. 364, Article ID 112355, 2020.

[9] F. Arrigoni, "Deterministic approximation of a stochastic metapopulation model," Advances in Applied Probability, vol. 35, no. 3, pp. 691-720, 2003.

[10] A. D. Barbour and A. Pugliese, "Asymptotic behavior of a metapopulation model, Advances," Annals of Applied. Probablity, vol. 15, no. 2, pp. 1306-1338, 2005.

[11] J. M. Ball, J. Carr, and O. Penrose, "The becker-döring cluster equations: basic properties and asymptotic behaviour of solutions," Communications in Mathematical Physics, vol. 104, no. 4, pp. 657-692, 1986.

[12] J. Carr and R. M. Dunwell, "Asymptotic behaviour of solutions to the becker-döring equations," Proceedings of the Edinburgh Mathematical Society, vol. 42, no. 2, pp. 415-424, 1999.

[13] G. I. Ibragimov, "The optimal pursuit problem reduced to an infinite system of differential equations," Journal of Applied Mathematics and Mechanics, vol. 77, no. 5, pp. 470-476, 2013.

[14] K. Deimling, Nonlinear Functional Analysis, Springer, Berlin, Germany, 1985.

[15] A. Salem, H. M. Alshehri, and L. Almaghamsi, "Measure of noncompactness for an infinite system of fractional langevin equation in a sequence space," Advances in Difference Equations, vol. 2021, p. 132, 2021.

[16] A. Salem and N. Mshary, "On the existence and uniqueness of solution to fractional-order langevin equation," Advances in Mathematical Physics, vol. 2020, Article ID 8890575, 11 pages, 2020.

[17] A. Salem and B. Alghamdi, "Multi-strip and multi-point boundary conditions for fractional langevin equation," Fractal and Fractional, vol. 4, no. 2, 18 pages, 2020.

[18] A. Salem and M. Alnegga, "Measure of noncompactness for hybrid langevin fractional differential equations," Axioms, vol. 9, no. 2, p. 59, 2020.

[19] A. Salem and A. Al-dosari, "Existence results of solution for fractional sturm-liouville inclusion involving composition with multi-maps," Journal of Taibah University for Science, vol. 14, no. 1, pp. 721-733, 2020.

[20] A. Salem, F. Alzahrani, and A. Al-dosari, "Attainabillity to solve fractional differential inclusion on the half line at resonance," Complexity, vol. 2020, Article ID 9609108, 13 pages, 2020.

[21] H. Baghani and J. J. Nieto, "on fractional langevin equation involving two fractional orders in different intervals," Nonlinear Analysis: Modelling and Control, vol. 24, pp. 884-897, 2019.

[22] R. P. Agarwal, A. Alsaedi, N. Alghamdi, S. K. Ntouyas, and B. Ahmad, "Existence results for multi-term fractional differential equations with nonlocal multi-point and multi-strip boundary conditions," Advances in Difference Equations.vol. 342, 2018.

[23] A. Salem, "Existence results of solutions for anti-periodic fractional langevin equation," Journal of Applied Analysis \& Computation, vol. 10, no. 6, pp. 2557-2574, 2020.

[24] A. Salem, F. Alzahrani, and M. Alnegga, "Coupled system of non-linear fractional langevin equations with multi-point and nonlocal integral boundary conditions," Mathematical. Problem in Engineering, vol. 2020, Article ID 7345658, 15 pages, 2020.

[25] A. Salem, F. Alzahrani, and B. Alghamdi, "Langevin equation involving two fractional orders with three-point boundary conditions," Differential and Integral Equations, vol. 33, pp. 163-180, 2020.

[26] A. Salem and M. Alnegga, "Fractional langevin equations with multi-point and non-local integral boundary conditions," Cogent Mathematics \& Statistics, vol. 7, no. 1, 2020.

[27] A. Salem, F. Alzahrani, and L. Almghamsi, "Fractional langevin equations with nonlocal integral boundary conditions," Mathematics, vol. 7, no. 5, pp. 1-10, 2019.

[28] A. Salem and B. Alghamdi, "Multi-point and anti-periodic conditions for generalized langevin equation with two fractional orders," Fractal and Fractional, vol. 3, no. 4, pp. 1-14, 2019.

[29] R. Bhatia, Notes in Functional Analysis, Hindustan Books Agency, New Delhi, India, 2009.

[30] I. E. Leonard, "Banach sequence spaces," Journal of Mathematical Analysis and Applications, vol. 54, no. 1, pp. 245-265, 1976.

[31] C. Chidume, Geometric Properties of Banach Spaces and Nonlinear Lterations, Springer-Verlag, Berlin, Germany, 1965.

[32] V. Georgi and Smirnov, Introduction To The Theory Of Differential Equations, p. 41, American Mathematical Society, Rhode Island, USA, 2002.

[33] J. P. Aubin and A. Cellina, Differential InclusionSet-Valued Maps and Viability Theory, Spring-Verlag, Berlin, Germany, 1984.

[34] J. P. Aubin and H. Frankowska, Set-Valued Analysis,Birkhauser, Bosten, MA, USA, 1990.

[35] K. Deimling, Multi-Valued Differential Equations, Walter De Gruyter, Berlin, Germany, 2011.

[36] C. Shiaw, K. K. Tan, and C. S. Wong, "Quasi-nonexpansive multi-valued maps and selections," Fundamental Mathematica, vol. 87, pp. 109-119, 1975.

[37] P. E. Howard, "Definitions of compact," Journal of Symbolic Logic, vol. 55, no. 2, pp. 645-655, 1990.

[38] A. Atangana and D. Baleanu, "New fractional derivatives with nonlocal and non-singular kernel: theory and application to heat transfer model," Thermal Science, vol. 20, no. 2, pp. 763-769, 2016.

[39] M. Al-Refai, "Fractional differential equations involving caputo fractional derivative with mittag-leffler non-singular kernel: comparison principles and applications," Electronic Journal of Differential Equations, vol. 2018, no. 36, 2017.

[40] D. Baleanu and A. Fernandez, "On some new properties of fractional derivatives with mittag-leffler kernel," Communications in Nonlinear Science and Numerical Simulation, vol. 59, pp. 444-462, 2018.

[41] D. G. Prakasha, P. Veeresha, and J. Singh, "Fractional approach for equation descriping the water transport in unsaturated porous media with mittag-leffler kernel," Frontiers in Physics, vol. 7, no. 193, 2019. 
[42] J. Hristov, On the Atangana-Baleanu Drivative and its Relation to Fading Memory Concept: The Diffusion Equation Formulation, pp. 175-193, Springer Nature, Cham, Switzerland, 2019.

[43] F. Ali, S. Murtaza, N. A. Sheikh, and K. S. Nisar, "Atanganabaleanu fractional model for the flow of jeffrey nanofluid with diffusion thermo effects: applications in engine oil," Advances in Difference Equations, vol. 2019, 2019.

[44] T. C. Lim, "A fixed point theorem for multi-valued nonexpansive mappings in uniformaly convex banch space," Bullten of the American Mathematical Society, vol. 80, no. 6, pp. 1123-1126, 1974.

[45] A. Bunyawat and S. Suantai, "Common fixed points of a countable family of multivalued quasi-nonexpansive mappings in uniformly convex banach spaces," International Journal of Computer Mathematics, vol. 89, no. 16, pp. 22742279, 2012.

[46] C. Castaing and M. Valadier, Convex Analysis and Measurable Multifunctions, Springer, New York, NY, USA, 2006.

[47] S. Hu and N. S. Papageorgiou, Handbook of Multivalued Analysis, Kluwer Dordrecht, Dordrecht, The Netherlands, 1997. 